\title{
Laparoscopic management of ruptured heterotopic pregnancy after intrauterine insemination
}

\author{
Marie Hutchinson MD, Cynthia Chan MD MScHQ
}

$\mathrm{A}$ 29-year-old gravida 3 para 1 woman (gestational age 12 weeks, 4 days) was referred from a peripheral hospital for worsening abdominal pain in the right lower quadrant. Two early ultrasound examinations (at six and nine weeks) had confirmed dates and viability of the fetus, showing no adnexal masses. The patient had undergone a previous cesarean section at term and a spontaneous abortion at eight weeks gestational age. All pregnancies had been conceived with intrauterine insemination, without the need for ovulation induction. Before the current intrauterine insemination cycle, the patient had two follicles, one on each side. The couple's infertility was secondary to male factor infertility and polycystic ovarian syndrome; hysterosalpingography had shown bilateral patency. She had no history of sexually transmitted infections or endometriosis. She had a history of obesity and laparoscopic cholecystectomy.

The patient had presented to her local emergency department one week earlier, with a threeday history of right lower quadrant pain. Ultrasonography at that time showed a right ovarian cyst measuring $3.8 \times 1.8 \times 2.1 \mathrm{~cm}$, consistent with a hemorrhagic or corpus luteal cyst, with no free fluid. The appendix was not visualized. Mild right hydronephrosis was noted, with no renal stones. The pain resolved, and the patient was discharged home.

One week later, the patient went back to her local emergency department with acute recurrence of pain. Focal peritonitis was noted in the right lower quadrant. Bimanual examination showed tenderness of the right adnexae, but no palpable mass. The vital signs were normal. Repeat ultrasonography showed mild free fluid with an enlarged, edematous right ovary measuring $5.7 \times$ $4.4 \times 4.6 \mathrm{~cm}$, with minimal flow (Figure 1). A viable intrauterine pregnancy was found, with no hydronephrosis or renal stones. Blood work showed a leukocyte count of 11.9 (normal 4.0 10.0) $\times 10^{3} / \mu \mathrm{L}$, hemoglobin 127 (normal 12.315.7) $\mathrm{g} / \mathrm{L}$ and human chorionic gonadotropin 69284 (normal [nonpregnancy] < 5) IU/L. The results of urine culture were negative.
The patient was transferred to our tertiary centre for possible surgical management. Upon arrival, she reported pain of the shoulder tip and shortness of breath. She had tachycardia (110 beats/min) and required ongoing opioid analgesia for pain. The right lower quadrant was focally peritonitic. Repeat blood work showed a drop in hemoglobin, to $99 \mathrm{~g} / \mathrm{L}$. We decided to proceed with laparoscopy for suspected ovarian torsion or ruptured cyst; heterotopic pregnancy was thought to be less likely, given the previously normal ultrasonography findings. The patient was informed of the risks of surgical management to her intrauterine pregnancy, as well as the possibility of oophorectomy or salpingectomy, which could affect her future fertility.

Given the patient's previous surgical history and given that she was pregnant, we performed laparoscopic entry in the left upper quadrant (Palmer point), following decompression of the stomach with an oral-gastric tube. Inspection of the pelvis showed no adhesions, but there was moderate hemoperitoneum with a gravid uterus and normal left ovary and tube to the fimbriated end (Figure 2). The right ovary was normal; however, a gestational sac within a hematosalpinx was noted, with active bleeding from its rupture site at the ampulla (Figure 3). Right salpingectomy was performed without complications. The estimated blood loss was $300 \mathrm{~mL}$, with most of this quantity having been noted upon entry.

The patient was discharged home the next morning, with hemoglobin stable at $100 \mathrm{~g} / \mathrm{L}$.

\section{KEY POINTS}

- Heterotopic pregnancy should always be considered in cases of unilateral abdominal pain occurring in pregnancy achieved through assisted reproductive technologies.

- Clinical suspicion may supersede negative imaging results, because ultrasonography may not be able to show a heterotopic pregnancy.

- Once heterotopic pregnancy is diagnosed, prompt surgical management is essential to allow continuation of the desired uterine pregnancy and to prevent maternal morbidity or death.

- Regardless of how the heterotopic pregnancy is managed operatively, the intrauterine pregnancy is at increased risk of loss. 
Pathologic examination confirmed the presence of a gestational sac within the ruptured right fallopian tube, with no signs of infection. Fetal nuchal translucency ultrasonography 10 days later confirmed continued live gestation.

\section{Discussion}

Ectopic pregnancy, in which a fertilized ovum implants outside of the endometrial cavity, remains one of the leading causes of first-

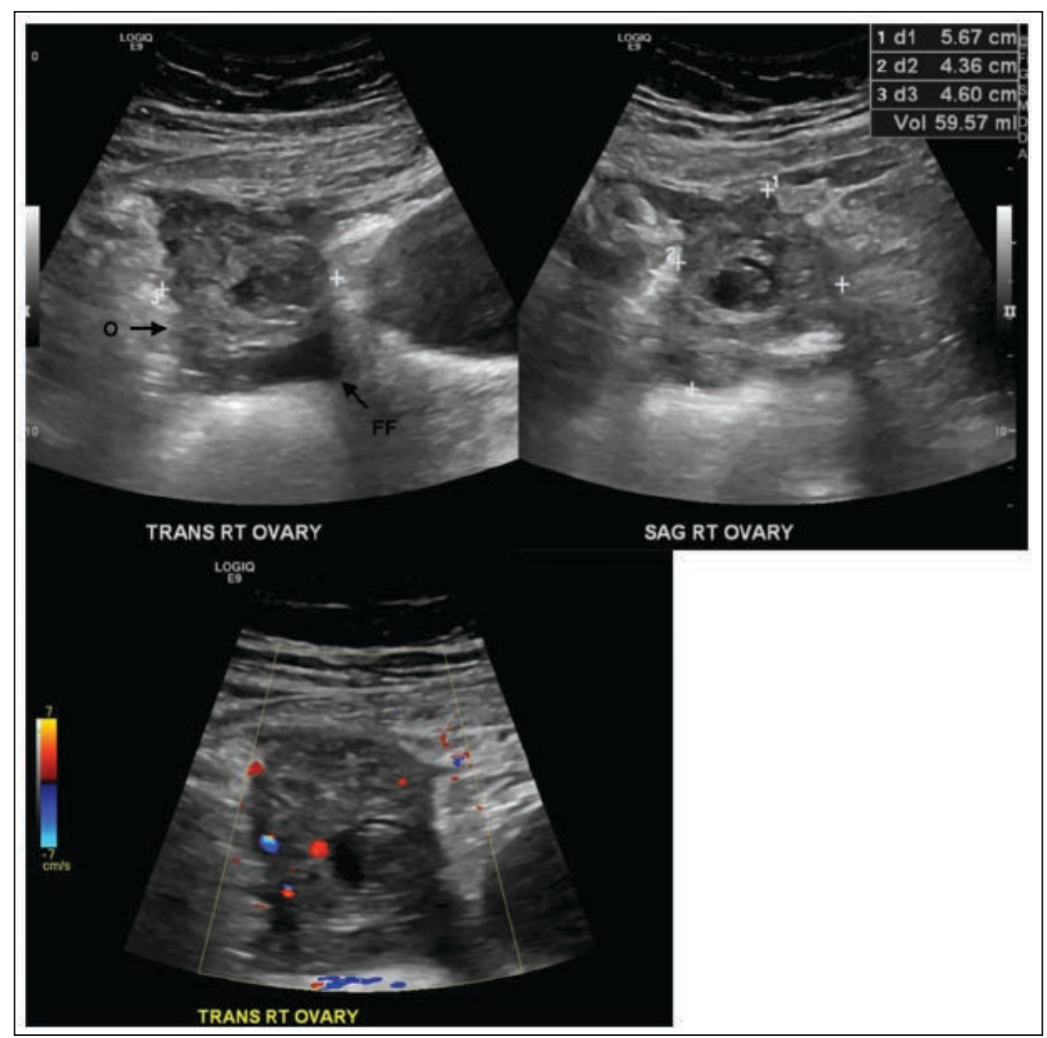

Figure 1: Preoperative ultrasound images in a 29-year-old gravida 3 para 1 woman (gestational age 12 weeks, 4 days) with abdominal pain, showing the enlarged edematous right ovary (0), which measured $5.7 \times 4.4 \times 4.6 \mathrm{~cm}$. Mild free fluid (FF) is also evident within the pelvis. Colour Doppler imaging showed minimal blood flow within the ovary, which suggested intermittent ovarian torsion.

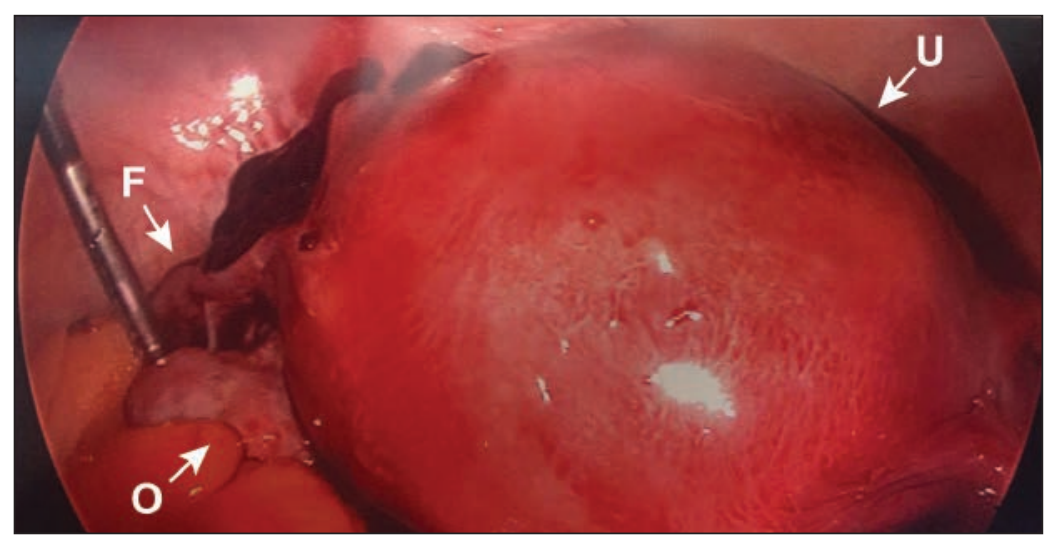

Figure 2: Laparoscopic image showing the gravid uterus $(U)$, the normal left fallopian tube (F) and the normal left ovary (O), surrounded by moderate hemoperitoneum. trimester maternal morbidity and mortality in the developed world. ${ }^{1,2}$ After natural conception, the concurrent presence of an ectopic pregnancy and an intrauterine pregnancy, known as heterotopic pregnancy, is rare (incidence 1 in 30000$).^{3}$ The use of assisted reproductive technologies, such as in vitro fertilization and intrauterine insemination, substantially increases the incidence of heterotopic pregnancy, to 1 in $125 .^{3}$ The embryo may be located within the fallopian tube $(90 \%$ of cases), an ovary, the cervix, the abdomen or a previous cesarean section scar. ${ }^{1}$ Diagnosis often occurs at less than eight weeks gestational age through the routine ultrasonography screening and close clinical follow-up that usually occur with assisted reproductive technologies. ${ }^{3,4}$ Tubal cases tend to be diagnosed earlier, because the confined space results in earlier pain or rupture. ${ }^{5}$ Seldom is heterotopic pregnancy discovered nearing or as late as the second trimester.

The diagnosis of heterotopic pregnancy can be challenging, because the traditional diagnostic clues to ectopic pregnancy are misleading in this situation. Classically, an inappropriately low increase in human chorionic gonadotropin leads to diagnosis of an ectopic pregnancy, but a concurrent pregnancy makes the value difficult to interpret. Health care providers are also often falsely reassured by ultrasonography findings showing an intrauterine pregnancy, and they may fail to consider an additional ectopic pregnancy, given its rarity.

In a large case series review, only $26 \%$ of cases were confirmed by ultrasonography, with the remainder confirmed surgically. ${ }^{6}$ This delay in diagnosis was reflected in a large proportion of patients presenting with hemoperitoneum (50\%) and hemorrhagic shock (20\%). Early ultrasonography during fertility treatment helps in assessing for ectopic and heterotopic pregnancy, but may lead to a false sense of security and delay the diagnosis. Another complicating factor is the misdiagnosis of heterotopic pregnancy as corpus luteal cyst, as occurred in our patient. This is a common pitfall, as both conditions may exhibit the "ring of fire" sign on ultrasonography, as in other previously reported cases., ${ }^{7,8}$

Diagnosis may be further delayed by a lack of symptoms. In a series of 13 women with heterotopic pregnancy who had undergone different types of fertility assistance, 7 (54\%) of the patients were asymptomatic. ${ }^{4}$ The mean gestational age at time of diagnosis was eight weeks, consistent with other case series. ${ }^{3}$ The advanced gestational age in our case was unusual, as most cases involving rupture of heterotopic pregnancy occur much earlier, between seven and nine weeks gestational age. ${ }^{3,6}$ 
The use of assisted reproductive technology is well known to be associated with heterotopic pregnancy. However, most of these cases arise from in vitro fertilization or frozen embryo transfer for tubal factor infertility, with up to $1 \%$ of patients having an ectopic pregnancy. ${ }^{6}$ Heterotopic pregnancy after intrauterine insemination, with or without ovulation induction, has been reported only rarely, perhaps because most authors group the technique under the broader heading of assisted reproductive technology, without differentiating among methods. Heterotopic pregnancy after ovulation induction has a reported incidence of 33 per 10000 cases. ${ }^{9}$ However, the incidence after intrauterine insemination only, without ovulation induction, is lacking. A previously reported case of heterotopic pregnancy after ovulation induction with intrauterine insemination was treated by laparoscopic salpingectomy at eight weeks gestational age, following rupture. ${ }^{8}$ Most of the cases reported in association with intrauterine insemination appear to have a form of twin pregnancy, either intrauterine or bilateral tubal. ${ }^{9}$

\section{Management}

Medical management of ectopic pregnancy with methotrexate is contraindicated if there is a concurrent intrauterine pregnancy. Injection of a feticide, such as potassium chloride, under ultrasound guidance, has been reported, with good outcomes. ${ }^{1}$ However, this form of treatment is contraindicated in cases of ruptured ectopic pregnancy. Heterotopic pregnancies are primarily treated surgically. Surgical options for tubal heterotopic pregnancy include either laparoscopy or laparotomy for removal of the ectopic pregnancy, which may require salpingostomy or salpingectomy. Laparoscopic treatment was successful in a previous case series, with most patients (10 of 13) receiving salpingectomy. ${ }^{4}$ There was no difference between laparotomy and laparoscopic management with respect to outcomes of the intrauterine pregnancy. Laparoscopic management is preferred because of its less invasive nature, shorter hospitalization and better patient outcomes.

Although the delay in diagnosis of heterotopic pregnancy in this case (at 12 weeks, 4 days gestational age) was not ideal, it did allow the patient to nearly reach the second trimester, when operative intervention is associated with better pregnancy outcomes. Regardless of the surgical method used in cases of heterotopic pregnancy, the intrauterine pregnancy is at increased risk of loss, with $31 \%$ of patients in a large case series having spontaneous abortion of the intrauterine pregnancy after treatment of the heterotopic pregnancy..$^{10}$ It is important to counsel patients about the possibility of spontaneous loss of the intrauterine pregnancy, as

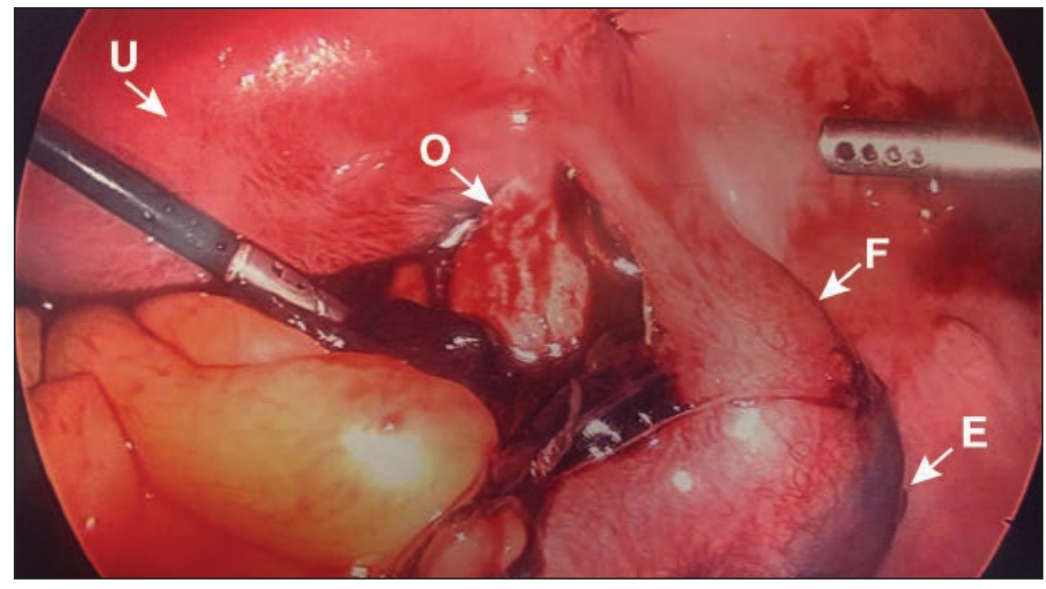

Figure 3: Laparoscopic image showing ruptured right heterotopic pregnancy (E) within the ampullary region of the fallopian tube $(F)$, beside the normal right ovary (0). U = uterus.

well as possible effects on future fertility, associated with the surgical intervention required for the affected adnexa.

\section{References}

1. Refaat B, Dalton E, Ledger WL. Ectopic pregnancy secondary to in vitro fertilisation-embryo transfer: pathogenic mechanisms and management strategies. Reprod Biol Endocrinol 2015;13:30.

2. Khan KS, Wojdyla D, Say L, et al. WHO analysis of causes of maternal death: a systematic review. Lancet 2006;367:1066-74.

3. Wang LL, Chen X, Ye D, et al. Misdiagnosis and delayed diagnosis for ectopic and heterotopic pregnancies after in vitro fertilization and embryo transfer. J Huazhong Univ Sci Technolog Med Sci 2014;34:103-7.

4. Louis-Sylvestre C, Morice P, Chapron C, et al. The role of laparoscopy in the diagnosis and management of heterotopic pregnancies. Hum Reprod 1997;12:1100-2.

5. Worley KC, Hnat MD, Cunningham FG. Advanced extrauterine pregnancy: diagnostic and therapeutic challenges. Am J Obstet Gynecol 2008;198:297.e1-7.

6. Barrenetxea G, Barinaga-Rementeria L, Lopez de Larruzea A, et al. Heterotopic pregnancy: two cases and a comparative review. Fertil Steril 2007;87:417.e9-15.

7. Talbot K, Simpson R, Price N, et al. Heterotopic pregnancy. J Obstet Gynaecol 2011;31:7-12.

8. Ikechebelu JI, Eleje GU. Heterotopic pregnancy following intrauterine insemination: successful management with salpingectomy and continuation of intrauterine pregnancy. Niger $J$ Clin Pract 2012;15:241-3.

9. Bugatto F, Quintero-Prado R, Kirk-Grohar J, et al. Heterotopic triplets: tubal ectopic and twin intrauterine pregnancy. A review of obstetric outcomes with a case report. Arch Gynecol Obstet 2010;282:601-6.

10. Clayton HB, Schieve LA, Peterson HB, et al. A comparison of heterotopic and intrauterine-only pregnancy outcomes after assisted reproductive technologies in the United States from 1999 to 2002. Fertil Steril 2007;87:303-9.

Affiliation: Department of Obstetrics and Gynaecology, Schulich School of Medicine and Dentistry, Western University, London, Ont.

Contributors: Cynthia Chan and Marie Hutchinson were involved in managing the patient's care, including surgical treatment. Marie Hutchinson drafted the manuscript, with final input and guidance from Cynthia Chan. Both of the authors approved the final version of the manuscript and agreed to act as guarantors of the work.

Acknowledgements: The authors would like to thank Debbie Penava for initiating care of the patient and providing editing assistance on the manuscript. They would also like to thank the staff at the London Health Sciences Centre who participated in this patient's care. 